\title{
A computational application for multi-skill nurse staffing in hospital units
}

Ana Respicio ${ }^{1,5^{*}}$ (D), Margarida Moz ${ }^{2}$, Margarida Vaz Pato ${ }^{2}$, Rute Somensi ${ }^{3}$ and Cecília Dias Flores ${ }^{4}$

\begin{abstract}
Background: Approaches to nurse staffing are commonly concerned with determining the minimum number of care hours according to the illness severity of patients. However, there is a gap in the literature considering multiskill and multi-shift nurse staffing. This study addresses nurse staffing per skill category, at a strategical decision level, by considering the organization of work in shifts and coping with variability in demand.

Methods: We developed a method to determine the nursing staff levels in a hospital, given the required patient assistance. This method relies on a new mathematical model for complying with the legislation and guidelines while minimizing salary costs. A spreadsheet-based tool was developed to embed the model and to allow simulating different scenarios and evaluating the impact of demand fluctuations, thus supporting decision-making on staff dimensioning.
\end{abstract}

Results: Experiments were carried out considering real data from a Brazilian hospital unit. The results obtained by the model support the current total staff level in the unit under study. However, the distribution of staff among different skill categories revealed that the current real situation can be improved.

Conclusions: The method allows the determining of staff level per shift and skill depending on the mix of patients' illness severity. Hospital management is offered the possibility of optimizing the staff level using a spreadsheet, a tool most managers are familiar with. In addition, it is possible to evaluate the implications of decisions on workforce dimensioning by simulating different demand scenarios. This tool can be easily adapted to other hospitals, using local rules and legislation.

Keywords: Nurse staffing, Multi-skill staffing, Shift work, Spreadsheet staffing model, Decision support

\section{Background}

Healthcare institutions must be well equipped with efficient and effective resources to provide healthcare to a more and more demanding population. As service must meet population needs, and costs with human resources represent a large amount of the operational costs in those institutions, accurate workforce planning is a very important task.

In the healthcare sector, nurses represent the main workforce to plan. The special kind of service they provide is to look after people in different severity conditions and

\footnotetext{
* Correspondence: alrespicio@fc.ul.pt

${ }^{1}$ CMAF-CIO, Faculdade de Ciências, Universidade de Lisboa, Lisbon, Portugal ${ }^{5}$ Departamento de Informática, Faculdade de Ciências, Universidade de Lisboa, Bloco C6, Piso 3, 1749-016 Lisboa, Portugal

Full list of author information is available at the end of the article
}

the care cannot be postponed. To determine the nurse staff levels, decision makers must take into account not only the variable demand for nursing care, but also the need to safeguard the health and goodwill of the nurses themselves, beyond the guidelines and legislation in force.

This section continues with a review of studies related to nurse workforce planning in general, and nurse staffing problems, in particular, highlighting some aspects that still deserve research. The definition of the multi-skill nurse staffing problem under study is then presented. Section Methods presents a two-phase methodology and describes the optimization model. An application using real-world data from a Brazilian hospital is described in Section Results, illustrated with the spreadsheet implementation of the model. Besides, a sensitivity analysis is conducted. A discussion of the experimental

(c) The Author(s). 2018 Open Access This article is distributed under the terms of the Creative Commons Attribution 4.0 International License (http://creativecommons.org/licenses/by/4.0/), which permits unrestricted use, distribution, and reproduction in any medium, provided you give appropriate credit to the original author(s) and the source, provide a link to the Creative Commons license, and indicate if changes were made. The Creative Commons Public Domain Dedication waiver (http://creativecommons.org/publicdomain/zero/1.0/) applies to the data made available in this article, unless otherwise stated. 
results follows in the corresponding section. The paper ends with the Section Conclusions.

\section{Nurse workforce planning}

In hospitals that work around the clock every day, the daily nurse work must be organized in shifts, and for each nurse a schedule must be posted in advance, indicating the sequence of days off and daily work shifts for the next planning period. Therefore, hospital administration needs to solve three interrelated problems, as stated in the seminal paper of Warner [1]. At the strategic decision level, the staffing problem consists of determining the dimension of the workforce required to provide the healthcare needed in all hospital units over a long-term planning horizon [2]. In the medium-term, at the tactical level, the scheduling/rostering problem is solved, so as to determine the set of schedules for all nurses of each unit that meets the variable demand per shift during a planning period, typically of one month, or 28 days, while respecting labour agreements $[1,3]$. In the short-term, at the operational decision level, the rerostering problem determines the necessary changes in the current roster when daily disruptions occur $[4,5]$.

These different types of decision have associated models that have been widely studied, however in the majority of the cases to tackle specific situations, as surveyed for human resources in general $[6,7]$. In the nurses' work planning context there are also some surveys revealing the considerable amount of research work in this area, which is mainly due to the specific legislation of each country and specific norms of the institutions [8-10]. Therefore, it is hard to conceive a general system for nurse staffing, scheduling/rostering and rerostering suitable for addressing all cases.

In the current study, we focus on the staffing problem with the purpose of determining the multi-skill nursing staff level per shift, derived from nurse-to-patient indicators applied to the demand for healthcare. We also take into account the further need of assigning nurses to shifts, complying with the labour legislation in force. Adequate nursing care is forced by hard constraints because it is the primary goal, while the minimization of salary costs is set as objective function.

We propose a new model to this problem and, relying on the model, we developed a spreadsheet-based tool that can be easily used in hospital units.

\section{Nurse staffing}

The nurse staffing problem must take into account nurses' workload measures and, in some cases, the allocation of nurses to the different daily shifts, considering the particular working environment, patient safety ratios and labour rules. Many authors highlight the positive correlation between the decrease of staffing levels and the decrease in quality of nurse care [11-13] as well as with the decrease in financial performance of hospitals [14]. Other authors also stress the negative impact of nurse turnover and the use of temporary nurses on patient care and nurse satisfaction [11, 15-17].

Hence, defining accurate nurse staffing levels is an important objective of every hospital administration, under pressure to reduce costs, while providing high quality health services. Approaches to nurse staff dimensioning are commonly concerned with determining the minimum number of nursing care hours per patient. Hurst [18] classified the methods to measure nursing workload into five categories: (1) professional judgement, based on the experience and knowledge of professionals; (2) average number of nurses per occupied bed; (3) the acuity-quality method, based on patient dependency; (4) regression analysis; and (5) the timed-task/activity method, based on the type and frequency of nursing interventions. Nursing workload naturally depends on the condition of patients, and several scoring systems to determine nurse-to-patient ratios have been proposed $[19,20]$. Therefore, hospitals and healthcare public administrations frequently lay down specific instructions to guide hospital managers and head nurses in staffing and scheduling tasks. That is the case of the "Resolução COFEN-293/2004" from the Brazilian Nurse Federal Council, which sets the nursing hours per patient for inpatient units according to four categories of illness severity [21].

Elkhuizen et al. [22] propose a general model that determines multi-shift nurse staff levels. The model does not consider different nursing skills and works for 24-h cover. It calls for computing average bed occupation on a daily basis, using calendar work days. Legal minima for nurse-to-patient ratios, set by international guidelines (USA and Australia), were used to establish the corresponding different ratios for three shifts (early, late and night). These ratios, together with the bed occupation and some coefficients to include absences, led to the advised number of nurses per shift. An application to a real setting is presented along with a what-if analysis. Kortbeek et al. [23] propose stochastic models for nurse staffing per shift with demand based on nurse-to-patient ratios. One of the models simultaneously staffs permanent nurses per hospital unit and floating nurses that may work at any hospital unit. The authors do not consider different nurse skills. They apply their models to a hospital in Amsterdam. To the best of our knowledge, the first work that explicitly models multi-skill multi-shift nurse staffing is that of Bordoloi and Weatherby [24], specifically developed for one hospital unit in Alaska. These authors present a linear programming model considering three skill categories and three shifts of equal length, thus defining nine decision variables. The demand per day (in hours) was estimated from historical data and divided per shifts according to the nurses' 
experience. The model minimizes salary costs and considers ratio constraints on work provided by differently-skilled staff and demand constraints per shift. These authors also perform a linear programming sensitivity analysis. More recently, Harper et al. [2] present a comprehensive study for multi-skill multi-shift nurse staffing in United Kingdom hospitals. The model proposed is a stochastic linear programming model that considers the minimization of costs aggregated over one year, subject to demand-fulfilment constraints per day and for different skills. The proposed computational tool is applied with user-defined parameters to obtain an aggregate level of staff.

Other authors integrate staffing and scheduling. Venkataraman and Brusco [25] propose a stochastic methodology that starts by solving a mixed integer linear programming model for a six-month period. This model has two integer variables (number of full-time and part-time nurses) and six continuous variables (overtime hours per month) and its solution is given as input to another mixed integer linear programming model for the scheduling problem. Different shifts and different skills are considered. More recently, Maenhout and Vanhoucke [26] also integrate nurse staffing and scheduling, applied to a hospital in Ghent. This study proposes a mixed integer linear programming model where the staffing decisions are represented by surplus and slack variables that account for under or over staffing of multi-skilled nurses per shift, with respect to the required number of nurses per ward, skill, day and shift.

No research work was found that addresses the nurse staffing problem by simultaneously considering the multi-skill staff levels required for different length shifts; respecting the scores of a scoring system as well as labour legislation, guidelines and internal institution rules; and balancing the number of employees per skill and per shift. Moreover, the literature lacks computational tools to dimension differently-skilled nursing staff, that are easy for practitioners to use, and adaptable to different hospital environments.

\section{Problem definition}

The study reported in this paper concerns a nurse staffing problem that aims to determine the size of nursing teams in a Brazilian hospital context. Personnel are grouped into two categories according to healthcare nursing skills: nurses - the group of the more skilled personnel; and nursing technicians - those who perform the auxiliary and basic care tasks, although professionally certified. In the hospital, apart from this permanent pool, dimensioned at the strategic decision level, there is also a floating pool of nurses and technicians, dimensioned at the tactical level, outside the scope of this study. The number of nurses should be at least a given proportion of the total staff, depending on the patient mix. Once assigned to a shift, nurses and technicians of this pool do not switch to another shift during the long-term planning horizon. Personnel assigned to night shift cannot work on consecutive days, due to labour legislation. Therefore, these personnel are grouped into odd night shift and even night shift workers. Moreover, the staff level in these two shifts must be equal, due to hospital rules.

The main objective of the nurse staffing problem is to determine the number of nurses and of nursing technicians to be assigned to the permanent pool of a hospital unit, complying with the demand for healthcare per shift and minimizing salary costs. The needs for healthcare service must be continuously satisfied by workers who are entitled to a specific number of days off and holidays, as well as absences. In this context, all types of absences are taken into account by the application of a Technical Safety Index (TSI) [21].

\section{Methods}

To determine the dimension of the permanent pool of nurses and nursing technicians for a hospital, the proposed methodology follows two phases, as illustrated by Fig. 1. The staffing is solved per hospital unit because each unit has different requirements in terms of nursing work, due to the different patient illness severity. For instance, the Intensive Care Unit (ICU), the Neurosurgery Unit, and the Cerebrovascular Accident Unit (CVA) typically treat high severity illness patients. In addition, the personnel of the permanent pool are assigned per hospital unit. The method considers the demand for a day since the guidelines stipulate the amount of daily nursing care.

\section{Phase I}

Phase I computes the required number of nursing care hours per day, according to the specific guidelines in use. For this computation, the necessary data are the estimated number of patients and their respective illness severity. For instance, the Brazilian COFEN guidelines [21] recommend a minimum number of nursing hours per patient, per day for each category of illness severity (minimal, intermediary, semi-intensive, or intensive care). In addition, the minimum ratio of nurses to nursing workers is also computed (as proposed by COFEN).

The output of Phase I is given as input to Phase II.

\section{Phase II}

Phase II proceeds with the dimensioning of personnel, additionally considering the following data from the institutional rules: the hourly salary costs per level of skill (nurses and technicians) and shift (morning, afternoon, odd and even nights), the shifts lengths, lower bounds for the staff per skill and shift, and parameters to 


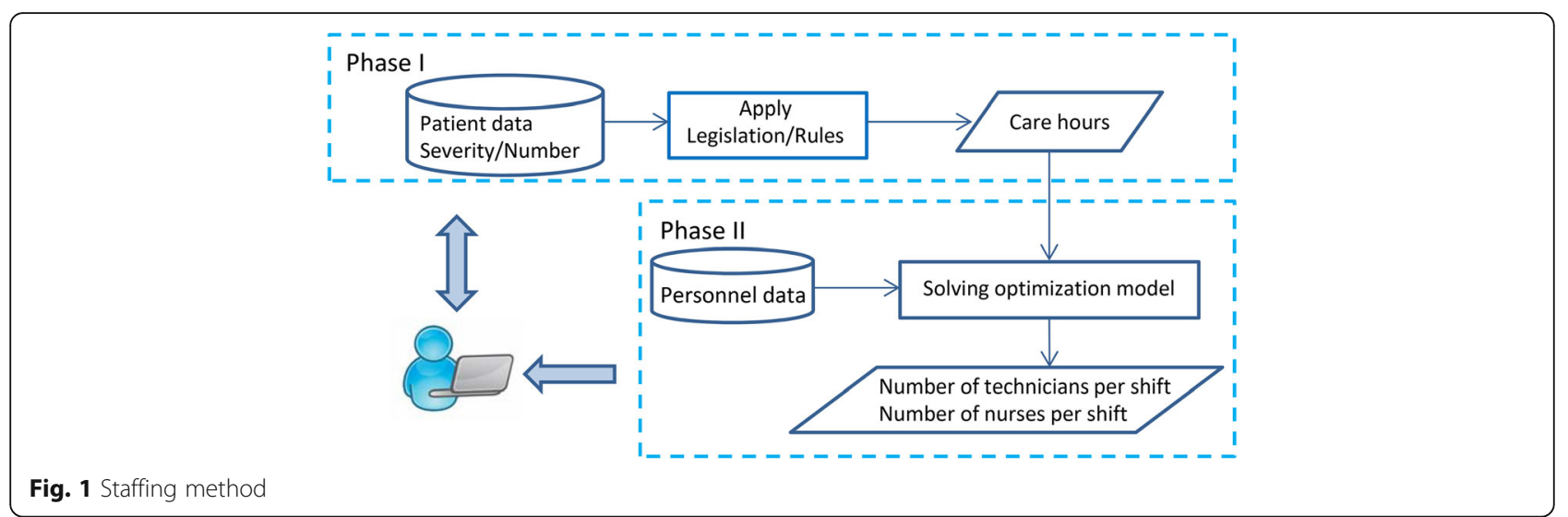

balance the staff between shifts and per skill. Other data come from COFEN guidelines: the TSI, acting as a buffer to cover all types of personnel absences, and the ratio of nurses to total staff.

The problem consists of determining the number of nurses and technicians required per shift, respecting the following conditions:

- the total number of hours of nurse and technician work assigned to all shifts cannot be less than the required nursing care hours per day (hours) [Constraints (1)];

- the percentage over the minimum staff level required to cover all types of personnel absences must be satisfied, corresponding to the application of the TSI (represented by the parameter $p$ ) [Constraint (2)];

- the number of nurses must be at least a given percentage-ratio (prop) of the total number of nursing-workers, i.e. nurses plus technicians [Constraint (3)];

- the number of workers, of each skill, assigned to morning and afternoon shifts do not differ more than a specified parameter, so to balance the workload among these shifts (represented by parameters $\alpha_{i}, i=n, t$ ) [Constraints (4)];

- two groups of night shifts are considered - odd nights (night 1) and even nights (night 2), because staff cannot work on consecutive nights, according to labour legislation [Constraints (5)];

- a minimum staff level per shift for each of nurses and technicians must be respected [Constraints (6)].

Adequate nursing care is enforced by the above hard constraints, while control of costs is achieved through the objective function of the model [Function (7)], where costs of both types of night shift are considered.

An optimization model is proposed and its mathematical formulation is presented in [Additional file 1]. It corresponds to an integer linear programming problem, insofar as it involves optimization of a linear function depending on non-negative integer variables subject to linear (in)equalities that express the constraints of the model. The solution for this model is obtained using a standard optimization solver.

The minimum imposed by Constraints (6) ensures that the demand to be immediately satisfied is covered, while Constraints (1) guarantee that these and other nursing activities, which do not need immediate execution in a given shift, are performed during the day. The minimum staff levels per shift can be adapted to accommodate different requirements for care per shift.

The decision maker can execute Phase I followed by Phase II repeatedly, with different demand scenarios to extract managerial indicators, thus supporting the decision making process regarding the staffing of the permanent pool. This allows him/her to evaluate the impact on staff level due to fluctuations in demand for healthcare. Complementarily, other constraints imposed by hospital management rules, sector guidelines, and legislation can be considered.

The results of Phase II - solution of the staffing problem - will be inputs for scheduling/rostering. In this hospital context, nursing-workers do not change shifts.

\section{Results}

The methodology is illustrated with the case of Hospital São José (HSJ). HSJ is part of Irmandade Santa Casa de Misericórdia de Porto Alegre, Brazil, and its units are characterized by the type of healthcare service provided: Emergency, Inpatient Units (ICU, CVA, PRT/CNV, and Neurosurgery), Intensive Care, Diagnosis and Treatment Units, and Outpatient and Surgical Center [27].

\section{Experiment description}

For the experiment, we considered real data from five HSJ units. Figure 2 displays the data used for model application. For each unit, data concerning the demand of 


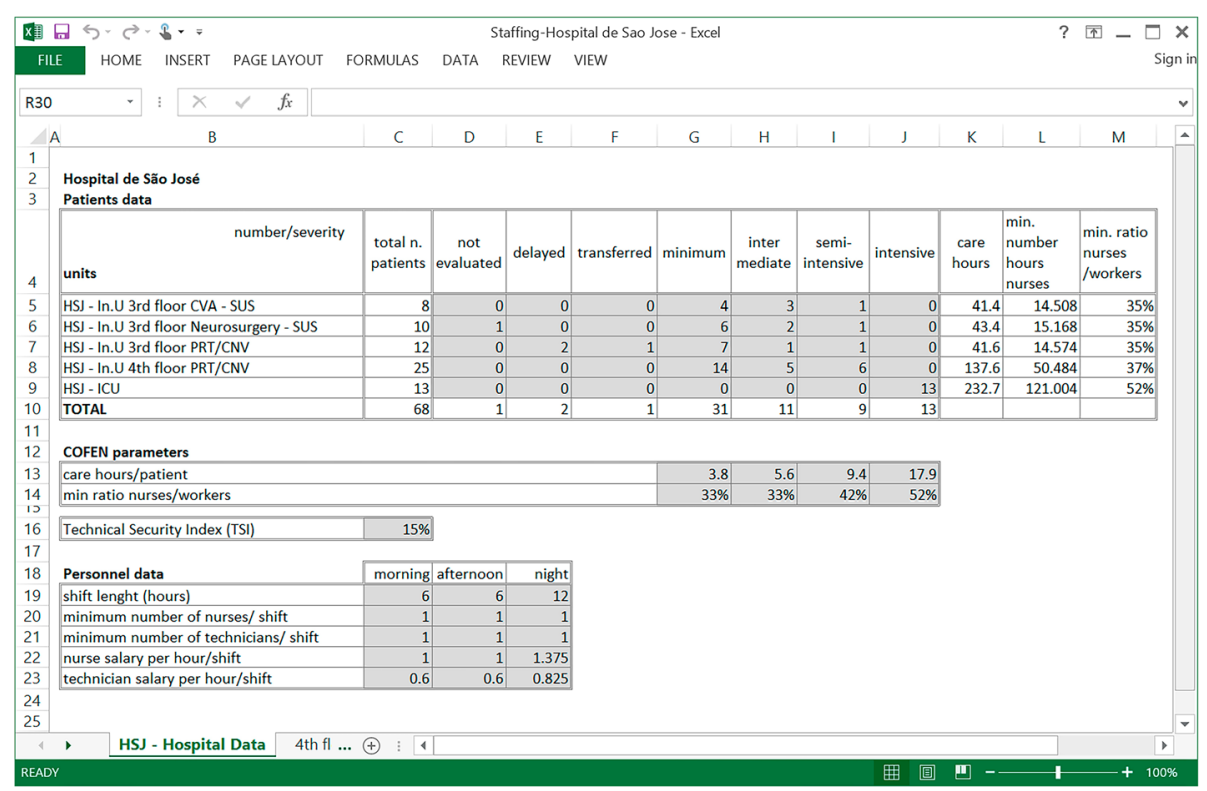

Fig. 2 Data used for model application

one day was considered, and the following numbers were compiled: number of inpatients requiring minimal care, intermediary care, semi-intensive care and intensive care. The unclassified inpatients were counted as needing intermediary care.

The Brazilian guidelines for nursing professional practice, COFEN [21], define the minimum number of nursing care hours per patient per $24 \mathrm{~h}$ (Art. 4th): $3.8 \mathrm{~h}$ per patient requiring minimal care, $5.6 \mathrm{~h}$ for intermediary care, $9.4 \mathrm{~h}$ for semi-intensive care and, $17.9 \mathrm{~h}$ for intensive care, respectively. COFEN also specifies a minimum ratio of nurses to the total workforce which is set according to the category with the largest number of inpatients. This proportion is set to $33 \%$ for the minimal and intermediary care, $42 \%$ for the semi-intensive care, and $52 \%$ for the intensive care. In addition, the TSI is set to $15 \%$, according to COFEN.

The method is applied using a Microsoft Excel spreadsheet [28]. The data in spreadsheet "HSJ Hospital Data" are organized in three tables (Fig. 2). The first table, "Patients data", contains the number of patients and their corresponding illness severity for the different hospital units on a specific day. The second table, "COFEN parameters", contains the model's parameters. Finally, the table "Personnel data" is used in Phase II. The shifts' lengths, which are not all equal, are consistent with the hospital operating rules. The minimum number of nurses and technicians per shift (lines 20 and 21) are set to one. Due to confidentiality reasons, the labour costs are omitted, and we assume a labour cost of 1 monetary unit per hour per nurse, and that the labour cost for technicians is
$60 \%$ of that value. Work in the night shift receives an increase of $37.5 \%$ in salary.

The output of Phase I, for each unit, is in column "care hours" and is given by summing up the product of the number of patients in the unit (columns $G$ to $J$ ) by the required care hours per patient severity (line 13), and also in column $M$, the ratio of nurses with respect to the total of nursing-workers.

\section{Spreadsheet implementation of the model}

The model for Phase II was implemented using the Solver from Excel [28] as shown in Fig. 3 for the unit "In. $4^{\text {th }}$ floor PRT/CNV". The spreadsheet " 4 th floor Mathematical Model" is organized into four main areas including all data, variables and formulae of the mathematical model in [Additional file 1].

The "nurses" area comprises columns $C$ to $E$ which include parameters (lines 3 to 11) and variables (line 12). Line 3, "hours constraint", contains the shifts lengths (in this case equal for all shifts and skills) taken from sheet "HSJ - Hospital Data". Line 5 "minimum percentage" contains the parameters needed for Constraint (3) of the problem by setting prop equal to the value in cell S5, which is equal to the minimum ratio of nurses to workers for this unit, in cell M8 of "HSJ - Hospital Data" sheet (Fig. 2). Line 10 contains the minimum staff level in each shift which, in this case, is set equal to 1 in Constraints (6) for all shifts. Line 11 has the salary costs per shift, which are computed by multiplying the value of labour cost per hour by the shift length in hours, using the data in spreadsheet "HSJ - Hospital Data". As 


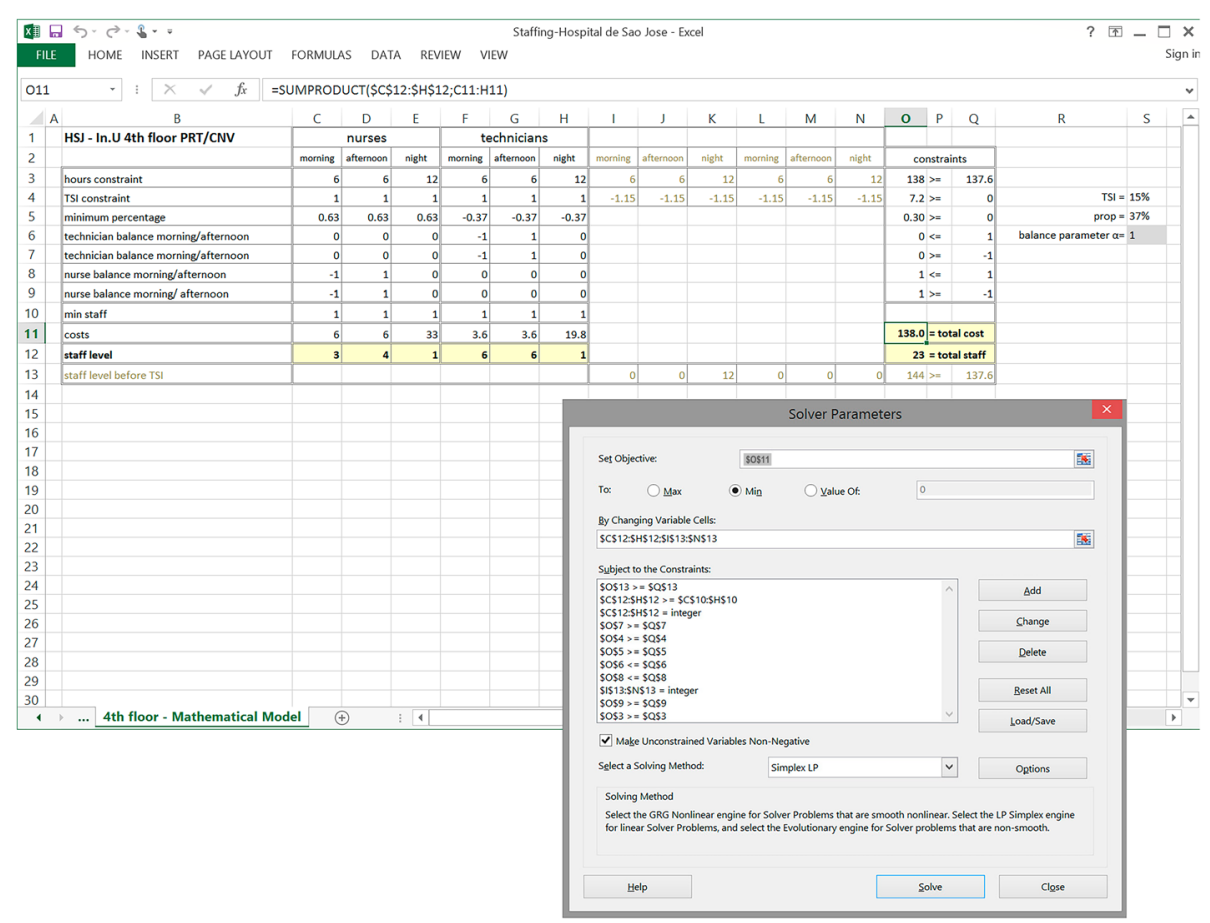

Fig. 3 Data and model results for Hospital São José, unit "In. $U 4^{\text {th }}$ floor PRT/CNV"

the model in the solver just refers to a single day, to take into account the staff for the two types of night shift, "night 1" and "night 2", the cost for the night shift is counted twice (Constraints (5)). Line 12 is reserved for the variables' values. For "technicians", the corresponding information is given in columns $F$ to $H$.

Columns $I$ to $N$ include data for auxiliary variables to respect staff levels before TSI application, which are used in Constraints (1) and (2): line 3 contains the shifts length to be included in the first inequalities of Constraints (1); line 4 implements Constraint (2), with TSI from "HSJ - Hospital Data", and line 13 contains the values of these variables.
Finally, columns $O$ to $Q$ include information for solving the problem: constraints and the objective function. Column $O$ (from lines 3 to 9 ) contains the excel formulae to be used in Solver, while column $Q$ contains the constant terms related to Constraints (1) to (4).

To balance the number of nurses and technicians between morning and afternoon shifts, a single parameter is set by the user in cell S6. In this application, we consider the balance parameter to be equal both for nurses and technicians.

The mathematical model is formulated for Solver software, in the window "Solver Parameters": in the field "Set Objective" cell $\mathrm{O} 11$ must be inserted. The respective value

Table 1 Impact of changes in parameters

\begin{tabular}{|c|c|c|c|c|c|c|c|c|c|c|c|c|c|}
\hline \multicolumn{2}{|l|}{ parameters } & \multicolumn{5}{|c|}{ staff level for nurses } & \multicolumn{5}{|c|}{$\underline{\text { staff level for technicians }}$} & \multirow[t]{2}{*}{ total staff } & \multirow[t]{2}{*}{ total cost } \\
\hline min. staff & $\begin{array}{l}\text { balance } \\
a_{n}=a_{t}\end{array}$ & morning & afternoon & night 1 & night 2 & total & mor-ning & afternoon & night 1 & night 2 & total & & \\
\hline 1 & 1 & 3 & 4 & 1 & 1 & 9 & 6 & 6 & 1 & 1 & 14 & 23 & 138.0 \\
\hline 1 & 2 & 4 & 3 & 1 & 1 & 9 & 5 & 7 & 1 & 1 & 14 & 23 & 138.0 \\
\hline 1 & 3 & 2 & 5 & 1 & 1 & 9 & 5 & 7 & 1 & 1 & 14 & 23 & 138.0 \\
\hline 2 & 1 & 2 & 3 & 2 & 2 & 9 & 5 & 5 & 2 & 2 & 14 & 23 & 171.6 \\
\hline 2 & 2 & 2 & 3 & 2 & 2 & 9 & 4 & 6 & 2 & 2 & 14 & 23 & 171.6 \\
\hline 2 & 3 & 2 & 3 & 2 & 2 & 9 & 4 & 6 & 2 & 2 & 14 & 23 & 171.6 \\
\hline 3 & 1 & 3 & 3 & 3 & 3 & 12 & 3 & 3 & 3 & 3 & 12 & 24 & 216.0 \\
\hline 3 & 2 & 3 & 3 & 3 & 3 & 12 & 3 & 3 & 3 & 3 & 12 & 24 & 216.0 \\
\hline 3 & 3 & 3 & 3 & 3 & 3 & 12 & 3 & 3 & 3 & 3 & 12 & 24 & 216.0 \\
\hline
\end{tabular}


is the objective function value to be minimized through the variables in C12:H12 and I13:N13. Constraints are added in the constraints section, all variables are set to nonnegative values and the method selected is "Simplex LP" (a simplex based branch-and-bound algorithm).

By clicking button "Solve", the algorithm runs and determines the model output, which is also shown in Fig. 3. The solution obtained, in cells C12 to H12, indicates the number of nurses and technicians for the three daily shifts. In this case, the solution gives 3 nurses for the morning, 4 for the afternoon and 1 for the night shifts; for technicians the numbers are 6,6 and 1 , respectively. The total salary cost for this solution is 138.0 monetary units, as shown in cell O11.

\section{Sensitivity of the model to parameter variation}

To assess the sensitivity of the model solutions regarding variations of the parameters, we analysed the impact of changes in the minimum staff level per

Table 2 Real data and model results for Hospital São José, in unit "In. U $4^{\text {th }}$ floor PRT/CNV"

\begin{tabular}{|c|c|c|c|c|c|c|c|c|c|c|c|c|}
\hline \multirow[t]{3}{*}{ date } & \multirow[t]{3}{*}{ weekday } & \multicolumn{8}{|l|}{ data } & \multicolumn{3}{|c|}{ results of Phase I } \\
\hline & & \multicolumn{8}{|c|}{ number of patients by illness severity level } & \multirow{2}{*}{$\begin{array}{l}\text { care hours } \\
\text { COFEN }\end{array}$} & \multirow{2}{*}{\multicolumn{2}{|c|}{$\begin{array}{l}\text { minimum ratio } \\
\text { nurses/workers } \\
\text { COFEN (\%) }\end{array}$}} \\
\hline & & \multicolumn{2}{|l|}{ total } & minimal & \multicolumn{2}{|c|}{ intermediary } & $\begin{array}{l}\text { semi- } \\
\text { intensive }\end{array}$ & \multicolumn{2}{|c|}{ intensive } & & & \\
\hline $\begin{array}{l}\text { 07-09- } \\
2016\end{array}$ & Wednesday & \multicolumn{2}{|l|}{34} & 21 & \multicolumn{2}{|l|}{10} & 3 & \multicolumn{2}{|l|}{0} & 164.0 & \multicolumn{2}{|l|}{35} \\
\hline $\begin{array}{l}\text { 08-09- } \\
2016\end{array}$ & Thursday & \multicolumn{2}{|l|}{25} & 15 & \multicolumn{2}{|l|}{8} & 2 & \multicolumn{2}{|l|}{0} & 120.6 & \multicolumn{2}{|c|}{34} \\
\hline $\begin{array}{l}\text { 09-09- } \\
2016\end{array}$ & Friday & \multicolumn{2}{|l|}{26} & 16 & \multicolumn{2}{|l|}{8} & 2 & \multicolumn{2}{|l|}{0} & 124.4 & \multicolumn{2}{|c|}{34} \\
\hline $\begin{array}{l}10-09- \\
2016\end{array}$ & Saturday & \multicolumn{2}{|c|}{21} & 13 & \multicolumn{2}{|l|}{7} & 1 & \multicolumn{2}{|l|}{0} & 98.0 & \multicolumn{2}{|c|}{34} \\
\hline $\begin{array}{l}11-09- \\
2016\end{array}$ & Sunday & \multicolumn{2}{|l|}{23} & 15 & \multicolumn{2}{|l|}{6} & 2 & \multicolumn{2}{|l|}{0} & 109.4 & \multicolumn{2}{|l|}{35} \\
\hline $\begin{array}{l}12-09- \\
2016\end{array}$ & Monday & \multicolumn{2}{|l|}{25} & 17 & 7 & & 1 & 0 & & 113.2 & 34 & \\
\hline $\begin{array}{l}13-09- \\
2016\end{array}$ & Tuesday & 34 & & 24 & 8 & & 2 & 0 & & 154.8 & 34 & \\
\hline $\begin{array}{l}14-09- \\
2016\end{array}$ & Wednesday & 35 & & 22 & 11 & & 2 & 0 & & 164.0 & 34 & \\
\hline staff leve & & & & & & & & & & & & \\
\hline date & results of $P h c$ & ase II & & & & & & & & & real situatic & \\
\hline & number of $n$ & urses per sh & & & number o & f technician & is per shift & & number & number of & number & number of \\
\hline & morning & afternoon & $\begin{array}{l}\text { night } \\
1\end{array}$ & night 2 & morning & afternoon & night 1 & $\begin{array}{l}\text { night } \\
2\end{array}$ & of nurses & technicians & of nurses & technicians \\
\hline $\begin{array}{l}07-09- \\
2016\end{array}$ & 3 & 4 & 2 & 2 & 7 & 8 & 1 & 1 & 11 & 17 & 4 & 22 \\
\hline $\begin{array}{l}08-09- \\
2016\end{array}$ & 3 & 3 & 1 & 1 & 5 & 6 & 1 & 1 & 8 & 13 & 4 & 22 \\
\hline $\begin{array}{l}\text { 09-09- } \\
2016\end{array}$ & 3 & 3 & 1 & 1 & 5 & 6 & 1 & 1 & 8 & 13 & 4 & 22 \\
\hline $\begin{array}{l}10-09- \\
2016\end{array}$ & 2 & 2 & 2 & 2 & 3 & 4 & 1 & 1 & 8 & 9 & 4 & 22 \\
\hline $\begin{array}{l}11-09- \\
2016\end{array}$ & 2 & 3 & 2 & 2 & 4 & 5 & 1 & 1 & 9 & 11 & 4 & 22 \\
\hline $\begin{array}{l}12-09- \\
2016\end{array}$ & 2 & 3 & 1 & 1 & 5 & 5 & 1 & 1 & 7 & 12 & 4 & 22 \\
\hline $\begin{array}{l}13-09- \\
2016\end{array}$ & 4 & 4 & 1 & 1 & 7 & 7 & 1 & 1 & 10 & 16 & 4 & 22 \\
\hline $\begin{array}{l}14-09- \\
2016\end{array}$ & 3 & 4 & 2 & 2 & 7 & 8 & 1 & 1 & 11 & 17 & 4 & 22 \\
\hline
\end{tabular}


shift along with variations of the balance parameter $\left(\alpha_{n}=\alpha_{t}\right)$. Table 1 presents the results of this analysis.

Increasing the minimum staff level required per shift increases the total cost because it forces the assignment of extra staff to more expensive shifts. Increasing the balance parameter provides more flexibility to distribute workers among shifts, and consequently the cost decreases. For instance, a solution for balance parameter $\alpha_{n}=2$, requiring a maximum difference of 2 nurses between the morning and afternoon shifts, is also a solution when $\alpha_{n}=3$ which requires a maximum difference of 3 workers between the morning and afternoon shifts, while a solution for $\alpha_{n}=3$ may not satisfy a balance parameter of $\alpha_{n}=2$. This can be observed in Table 1, when minimum staff is 1 , the solution for $\alpha_{n}=2$ includes 4 nurses in the morning and 3 in the afternoon, also satisfying $\alpha_{n}=3$; while the solution for $\alpha_{n}=3$ considers 2 nurses in the morning and 5 in the afternoon, and thus does not satisfy $\alpha_{n}=2$.

When the minimum number of staff is fixed, variations of the balance parameter value do not have an impact on the total cost or total number of staff. In fact, when the minimum number of staff is fixed to 1 , different solutions with the same cost were obtained for different values of the balance parameter (from 1 to 3 ). When the minimum staff level is set to 2 , the total cost increases by $24.2 \%$ and the staff levels remain equal to 9 for nurses and 14 for technicians. Increasing the minimum staff level from 2 to 3 increases the total cost by $25.8 \%$, leading to a solution where at least 12 nurses and 12 technicians must be assigned, which sum up to 24 (3 workers of each skill and shift).

\section{Discussion}

The methodology proposed was applied to data related with bed occupation and type of nursing care required by patients in several units of HSJ. In particular, a study was undertaken for the unit "In. U $4^{\text {th }}$ floor PRT/CNV", for a duration of eight consecutive days, from Wednesday, September 7, 2016. The first part of Table 2 displays the data relating to the number of patients by illness severity level, the number of nursing care hours according to COFEN (as explained in subsection "Hospital data") and the corresponding minimum ratio of nurses to workers (prop parameter in the model). The second part of the table presents the results obtained from Phase II of the method.

The results show that total staff required varies between 17 and 28 workers. The total staff in the real situation is constant and equal to 26. This number of workers is greater than or equal to the staff levels obtained by the method for all days except for Wednesdays, for which two additional workers are needed. Regarding the number of nurses, the results reveal that in the real situation there is a lack of nurses if the COFEN guidelines are applied, while the number of technicians is greater than the one obtained by the model. This reveals that in the current real situation the minimum ratio of nurses to the total workforce, which depends on the mix of patients' illness severity (subsection "Hospital data") is not covered by the permanent pool of nurses. In these cases, nurses from the floating pool may be assigned.

As a result of this experiment, we may observe that the proposed methodology was conceived to support the hospital management decision making process, so to improve the personnel dimensioning, and better agree with the COFEN guidelines.

\section{Conclusions}

A new methodology was developed to determine the multi-skill nursing staff level to be assigned per shift during a long-term planning horizon, in a hospital, complying with legislation, nurse work guidelines, and rules in force, while minimizing hospital costs. This complex staff dimensioning problem was adapted to the Brazilian context, considering a real world hospital, with two skill categories - nurses and technicians - and three work shifts of different durations. The proposed method applies to a single unit and allows the hospital management to determine the staff level per shift and per skill category as a function of the mix of severity. The workforce for a hospital can be obtained by executing the method for all its units.

The staff dimensioning method, at the strategic decision level, will provide input for lower level decision stages concerning personnel planning.

Another contribution of this study is a decision support tool, embedding the staffing method and implemented in a Microsoft Excel spreadsheet. [Additional file 2] contains the tool described in this manuscript. Most managers are familiar with usage of this software, therefore, the spreadsheet model is designed in an intelligible way to allow for an easy utilization. The tool also provides support to analyse scenarios with different mix of severity. Moreover, the decision maker can modify different parameters, such as shift lengths, salary costs, minimum staff level required per shift and skill, and the parameter to balance workforce, and evaluate their impact on results. The model and the tool developed are easily customizable to integrate the working rules of different hospital contexts.

As future work we intend to apply and evaluate the developed methodology in other healthcare contexts, namely in Portuguese public hospital units. 


\section{Additional files}

Additional file 1: Optimization model. The mathematical model used to represent the problem and to implement in the computational tool. (PDF $193 \mathrm{~kb}$ )

Additional file 2: Computational tool. The computational tool to support decision making on multi-skill nurse staffing in hospital units. (XLSX $19 \mathrm{~kb})$

\section{Abbreviations}

CVA: Cerebrovascular Accident Unit; HSJ: Hospital São José; ICU: Intensive Care Unit; PRT/CNV: Unit for Patients with Health Plan; SUS: Unified Health System (in Brazil)

\section{Acknowledgements}

We would like to thank the Hospital São José of the Irmandade Santa Casa de Misericórdia de Porto Alegre, Brazil for contributing with real data for the experiment. We acknowledge the reviewers for their helpful comments on a previous version of the manuscript. We thank Dr. Rob Mills for the English language review.

\section{Funding}

This work was partially supported by Portuguese funding from Fundação para a Ciência e a Tecnologia (FCT), under project UID/MAT/04561/2013 and Brazilian funding through project 442780/2014-1 - Edital MCTI/CNPq/Universal 14/2014. The funding body had no role in the design of the study and collection, analysis, and interpretation of data and in writing the manuscript.

\section{Availability of data and materials}

All data generated or analysed during this study are included in this article. The permission to access data was approved by the ethical in research committee "Comité de Ética em Pesquisa (CEP)" from Irmandade da Santa Casa de Misericórdia de Porto Alegre (ISCMPA) under registration CAAE: 44679015.0.0000.5335.

\section{Authors' contributions}

AR, MM, MVP are responsible for the methodology conception, implementation and design of the experiment. RS and CDF are responsible for problem definition, real data gathering and discussion. All authors contributed for the background section, read and approved the final manuscript.

\section{Ethics approval and consent to participate}

This study and the consent to collect and use the data was approved by the ethical in research committees "Comité de Ética em Pesquisa (CEP)" from Irmandade da Santa Casa de Misericórdia de Porto Alegre (ISCMPA) (https:// ensinoepesquisa.santacasa.org.br/comites-de-etica-em-pesquisa), under registration CAAE: 44679015.0.0000.5335 and "Comité de Ética em Pesquisa (CEP)" from Universidade Federal de Ciências da Saúde (UFCSPA) (https:// www.ufcspa.edu.br/index.php/cep/membros-do-comite) under registration CAAE: 44679015.0.3001.5345. The consent to participate was not required since no human's data are used.

\section{Competing interests}

The authors declare that they have no competing interests.

\section{Publisher's Note}

Springer Nature remains neutral with regard to jurisdictional claims in published maps and institutional affiliations.

\section{Author details}

${ }^{1} \mathrm{CMAF}-\mathrm{ClO}$, Faculdade de Ciências, Universidade de Lisboa, Lisbon, Portugal. ${ }^{2}$ ISEG and CMAF-CIO, Universidade de Lisboa, Lisbon, Portugal. ${ }^{3}$ Pavilhão Pereira Filho, Santa Casa de Misericórdia Porto Alegre and Universidade Federal de Ciências da Saúde de Porto Alegre, Hospital São José, Porto Alegre, Brazil. ${ }^{4}$ Universidade Federal de Ciências da Saúde de Porto Alegre, Porto Alegre, Brazil. ${ }^{5}$ Departamento de Informática, Faculdade de Ciências, Universidade de Lisboa, Bloco C6, Piso 3, 1749-016 Lisboa, Portugal.
Received: 29 August 2017 Accepted: 6 June 2018

Published online: 28 June 2018

\section{References}

1. Warner DM. Scheduling nursing personnel according to nursing preference: a mathematical programming approach. Oper Res. 1976;24(5):842-56.

2. Harper PR, Powell NH, Williams JE. Modelling the size and skill-mix of hospital nursing teams. J Oper Res Soc. 2010;61:768-79.

3. Smet $P$, Bilgin $B$, De Causmaecker $P$, Vanden Berghe $G$. Modelling and evaluation issues in nurse rostering. Ann Oper Res. 2014;218:303-26.

4. Moz M, Pato MV. A genetic algorithm approach to a nurse Rerostering problem. Computers OR. 2007;34:667-91.

5. Clark A, Moule P, Topping A, Serpell M. Rescheduling nursing shifts: scoping the challenge and examining the potential of mathematical model based tools. J Nursing Manag. 2015:23:411-20.

6. Van den Bergh J, Belien J, De Bruecker P, Demeulemeester E, De Boeck L. Personnel scheduling: a literature review. EJOR. 2013;226(3):367-85.

7. Defraeye $M, \operatorname{Van}$ Nieuwenhuyse I. Staffing and scheduling under nonstationary demand for service: a literature review. Omega. 2016;58:4-25.

8. Cheang B, Li H, Lim A, Rodrigues B. Nurse rostering problems - a bibliographic survey. EJOR. 2003;151(3):447-60.

9. Burke EK, De Causmaecker P, Berghe GV, Van Landeghem H. The state of the art of nurse rostering. J Scheduling. 2004;7(6):441-99.

10. Kellogg DL, Walczak S. Nurse scheduling: from academia to implementation or not? Interfaces. 2007;37(4):355-69.

11. Kash BA, Castle NG, Naufal GS, Hawes C. Effect of staff turnover on staffing: a closer look at registered nurses, licensed vocational nurses, and certified nursing assistants. The Gerontologist. 2006;46(5):609-19.

12. Rochefort $C M$, Buckeridge $\mathrm{DL}$, Abrahamowicz M. Improving patient safety by optimizing the use of nursing human resources. Implement Sci. 2015;10:89.

13. Giuliano KK, Danesh V, Funk M. The relationship between nurse staffing and 30-day readmission for adults with heart failure. J Nursing Admin. 2016; 46(1):25-9.

14. Everhart D, Neff D, Al-Amin M, Nogle J, Weech-Maldonado R. The effects of nurse staffing on hospital financial performance: competitive versus less competitive markets. Health Care Manag Rev. 2013;38(2):146-55.

15. Flynn M, Mckeown M. Nurse staffing levels revisited: a consideration of key issues in nurse staffing levels and skill mix research. J Nursing Manag. 2009;17(6):759-66.

16. O'Brien-Pallas L, Murphy GT, Shamian J, Li X, Hayes LJ. Impact and determinants of nurse turnover: a pan-Canadian study. J Nursing Manag. 2010;18:1073-86.

17. Chana N, Kennedy P, Chessell ZJ. Nursing staffs' emotional well-being and caring behaviours. J Clinical Nursing. 2015;24:2835-48.

18. Hurst K. Selecting and applying methods for estimating the size and mix of nursing teams: a systematic review of the literature commissioned by the Department of Health. Leeds: Nuffield Institute for Health, University of Leeds; 2003.

19. Cullen DJ, Civetta JM, Briggs BA, Ferrara LC. Therapeutic intervention scoring system: a method for quantitative comparison of patient care. Crit Care Med. 1974;2(2):57-112.

20. Miranda DR, Nap R, de Rijk A, Schaufeli W, lapichino G, et al. Nursing activities score. Crit Care Med. 2003:31(2):374-82.

21. COFEN. Resolução COFEN-293/2004. 2004. http://www.cofen.gov.br/resoluocofen-2932004 4329.html. Accessed 26 Feb 2016.

22. Elkhuizen SG, Bor G, Smeenk M, Klazinga NS, Bakker PJ. Capacity management of nursing staff as a vehicle for organizational improvement. BMC Health Serv Res. 2007;7(1):1.

23. Kortbeek N, Braaksma A, Burger CA, Bakker PJ, Boucherie RJ. Flexible nurse staffing based on hourly bed census predictions. Int J Prod Econ. 2015;161:167-80.

24. Bordoloi SK, Weatherby EJ. Managerial implications of calculating optimum nurse staffing in medical units. Health Care Man Rev. 1999:24(4):35-44.

25. Venkataraman R, Brusco MJ. An integrated analysis of nurse staffing and scheduling policies. Omega. 1996;24(1):57-71.

26. Maenhout B, Vanhoucke M. An integrated nurse staffing and scheduling analysis for longer-term nursing staff allocation problems. Omega. 2013; 41(2):485-99.

27. ISCMPA. Hospital São José - Neurosurgery, Irmandade da Santa Casa da Misericórdia de Porto Alegre. 2017. http://www.santacasa.org.br/en/sao-jose Accessed 10 Feb 2017.

28. Microsoft. Microsoft Office Professional Plus 2013. 2017. https:/technet. microsoft.com/ en-us/office/. Accessed 10 Feb 2017. 